\title{
WIMAN-VALIRON THEORY IN SEVERAL VARIABLES
}

\author{
P. C. Fenton \\ University of Otago, Department of Mathematics and Statistics \\ P.O. Box 56, Dunedin 9054, New Zealand; pfenton@maths.otago.ac.nz
}

\begin{abstract}
Wiman-Valiron theory of entire functions of several complex variables is developed up to results on the asymptotic behaviour of the derivatives.
\end{abstract}

\section{Introduction}

Let $f$ be a transcendental entire function of $n$ complex variables $z_{1}, \ldots, z_{n}$, with Taylor series

$$
f(\mathbf{z})=\sum_{\mathbf{j}} a_{\mathbf{j}} \mathbf{z}^{\mathbf{j}}
$$

where $\mathbf{z}=\left(z_{1}, \ldots, z_{n}\right), \mathbf{j}=\left(j_{1}, \ldots j_{n}\right), j_{1}, \ldots, j_{n}$ being non-negative integers, and $\mathbf{z}^{\mathbf{j}}=z_{1}^{j_{1}} \cdots z_{n}^{j_{n}}$. We adopt the conventions that $0^{0}=1$ and $0 / 0=1$. The aim here is to establish results for general $n$ that are known for $n=1$ and, in a somewhat piecemeal and unsystematic form, for $n=2[6,7,10]$.

In what follows, $\mathbf{0}=(0, \ldots, 0), \mathbf{1}=(1, \ldots, 1)$ and, for $1 \leq j \leq n, \mathbf{1}_{j}=$ $(0, \ldots, 1, \ldots, 0)$, the vector in which all components are 0 except the $j$ th, which is 1 . For $\mathbf{z}=\left(z_{1}, \ldots, z_{n}\right)$ and $\mathbf{w}=\left(w_{1}, \ldots, w_{n}\right)$, we write $\mathbf{z w}=\left(z_{1} w_{1}, \ldots, z_{n} w_{n}\right)$, $\mathbf{z} / \mathbf{w}=\left(z_{1} / w_{1}, \ldots, z_{n} / w_{n}\right), \mathbf{z} \cdot \mathbf{w}=z_{1} w_{1}+\cdots+z_{n} w_{n},|\mathbf{z}|=\left(\left|z_{1}\right|, \ldots,\left|z_{n}\right|\right),\|\mathbf{z}\|=$ $\max _{1 \leq j \leq n}\left|z_{j}\right|$ and $\|\mathbf{z}\|_{\circ}=\left|z_{1}\right|+\cdots+\left|z_{n}\right|$. We call $\mathbf{j}$ the degree of $a_{\mathbf{j}} \mathbf{z}^{\mathbf{j}}$, and write $\mathbf{j}=\operatorname{deg}\left(a_{\mathbf{j}} \mathbf{z}^{\mathbf{j}}\right)$.

With the understanding that $\mathbf{r} \geq \mathbf{s}$ or $\mathbf{r}>\mathbf{s}$ means $r_{j} \geq s_{j}$ or $r_{j}>s_{j}$ for $1 \leq j \leq n$, we write $\log \mathbf{r}=\left(\log r_{1}, \ldots, \log r_{n}\right)$ for $\mathbf{r}>\mathbf{0}$. More generally, if $h$ is a real- or complex-valued function of a real or complex variable $z$, we define $\mathbf{h}(\mathbf{z})=$ $\left(h\left(z_{1}\right), \ldots, h\left(z_{n}\right)\right)$. We write $\log \mathbf{S}=\{\log \mathbf{r}: \mathbf{r} \in \mathbf{S}\}$ for any set $\mathbf{S} \subseteq\{\mathbf{r}: \mathbf{r}>\mathbf{0}\}$, and define the logarithmic measure of $\mathbf{S}$ by

$$
\operatorname{logmeas} \mathbf{S}=\int_{\log \mathbf{S}} d \mathbf{r}
$$

where $d \mathbf{r}=d r_{1} \cdots d r_{n}$. Given $\mathbf{r}_{2} \geq \mathbf{r}_{1} \geq \mathbf{0}$, we write $\Delta_{\mathbf{r}_{1}, \mathbf{r}_{2}}=\left\{\mathbf{r}: \mathbf{r}_{1}<\mathbf{r}<\mathbf{r}_{2}\right\}$.

For $\mathbf{r} \geq \mathbf{0}$, the maximum term of the function $f$ given by (1) is

$$
\mu(\mathbf{r}, f)=\max _{\mathbf{j} \geq \mathbf{0}}\left|a_{\mathbf{j}}\right| \mathbf{r}^{\mathbf{j}}
$$

this is well-defined since, for each fixed $\mathbf{r}, a_{\mathbf{j}} \mathbf{r}^{\mathbf{j}} \rightarrow 0$ as $\|\mathbf{j}\| \rightarrow \infty$. Any $\mathbf{j}$ for which the maximum in (3) is attained is a central index of $f$, denoted by $\mathbf{N}(\mathbf{r}, f)$. The central index is uniquely defined for most $\mathbf{r} \geq \mathbf{0}$, as we will see. We also define the maximum modulus of $f$ for $\mathbf{r} \geq \mathbf{0}$ by $M(\mathbf{r}, f)=\max _{|\mathbf{z}|=\mathbf{r}}|f(\mathbf{z})|$.

doi:10.5186/aasfm.2013.3804

2010 Mathematics Subject Classification: Primary 30D15.

Key words: Wiman-Valiron, entire, several variables. 
These definitions apply equally to functions that are analytic at $\mathbf{0}$ but not necessarily entire. We will call a function $h$ that is analytic in $\|\mathbf{z}\|<R$ fully indexed if, given any $\mathbf{N} \in(\mathbb{N} \cup\{0\})^{n}$, we can find $\mathbf{r}_{\mathbf{N}}$, with $0<\left\|\mathbf{r}_{\mathbf{N}}\right\|<R$, such that the central index of $h$ at $\mathbf{r}_{\mathbf{N}}$ is unique and equal to $\mathbf{N}$. We call $\left\{\mathbf{r}_{\mathbf{N}}: \mathbf{N} \in(\mathbb{N} \cup\{0\})^{n}\right\}$ an indexing set for $h$.

Given an increasing function $\alpha:[0, \infty) \rightarrow \mathbb{R}$, we define

$$
\alpha_{\mathbf{j}}=\alpha_{j_{1}} \cdots \alpha_{j_{n}}, \text { where } \alpha_{j}=\exp \left(-\int_{0}^{j} \alpha(t) d t\right),
$$

and

$$
\boldsymbol{\rho}_{\mathbf{j}}=\left(\rho_{j_{1}}, \ldots, \rho_{j_{n}}\right) \text {, where } \rho_{j}=\exp (\alpha(j)) .
$$

It can be checked that, for all $\mathbf{j} \geq \mathbf{0}$ and $\mathbf{N} \geq \mathbf{0}$,

$$
\alpha_{\mathbf{j}} \rho_{\mathbf{N}}^{\mathbf{j}}<\alpha_{\mathbf{N}} \rho_{\mathbf{N}}^{\mathbf{N}}(\mathbf{j} \neq \mathbf{N}) \text {. }
$$

Thus

$$
h_{\alpha}(z)=\sum_{\mathbf{j} \geq \mathbf{0}} \alpha_{\mathbf{j}} \mathbf{z}^{\mathbf{j}}
$$

which is analytic in $\|\mathbf{z}\|<e^{\alpha_{\infty}}$, where $\alpha_{\infty}=\lim _{t \rightarrow \infty} \alpha(t)$, is fully indexed and $\left\{\boldsymbol{\rho}_{\mathbf{N}}: \mathbf{N} \in(\mathbb{N} \cup\{0\})^{n}\right\}$ is an indexing set for $h_{\alpha}$.

The sequences $\alpha_{j}$ and $\rho_{j}$ and the function $h_{\alpha}$ are elements of the comparison version of Wiman-Valiron theory developed by Kövari [11, 12], Clunie [2, 3], Hayman $[9,10]$ and others. Hayman's survey [10] gives a detailed account of this method, as well as a comprehensive list of references, and his approach is carried over into what follows. A reader familiar with [10] will be aware of the debt that I owe it.

Our methods apply generally to all entire functions but the main inequalities (the subject of Theorem 1 below) can be applied to give more refined results for, for example, functions of finite order. This has not been pursued. In the one-variable case, there is a version of the theory that gives results for functions of finite lower growth $[4,5]$. A similar approach appears to be possible in several variables but is beyond the scope of this note, and in any case would be of secondary interest.

The results presented here are known, in less precise forms, in the one- and two-variable cases, and the narrative direction, so to speak, is predictable. However, geometrical intuition, which bolsters arguments in one and two variables, is not available in higher dimensions and then the discussion, especially of the central index (in section 3 below), needs to be undertaken more formally. Theorem 1 gives inequalities for the terms of the series (1) on the so-called normal set. For $n=2$ it is more general than its equivalent in [6], and when $n=1$ gives the one-variable inequalities in [10, p. $319 \mathrm{ff}$.]. It also provides a better estimate of the size of the normal set than the one given in [6], which has the useful consequence that conclusions about the growth of solutions of partial differential equations, which in [8] appear as lower estimates for the order, can be improved to give lower estimates for the lower order. Theorem 2 expresses the fact that, at normal values, the terms of the series (1) that are far away from the maximum term are collectively insignificant in comparison with the maximum term. This is needed to establish the asymptotic expressions for the derivatives of $f$, which are the concern of Theorem 3. The statement of Theorem 3 is more general than its two-variable counterpart in [7]; in particular, the expressions for the derivatives apply in discs of a specified radius about 'near maximum' points rather 
than simply at the maximum points themselves. Theorem 3 has the disadvantage that it contains a hypothesis - which cannot be eliminated, as we will show - that makes it difficult to apply in practice. This disadvantage can be circumvented using Theorem 4, which involves a transformed version of $f$. The idea is to use Theorem 4 to obtain information about the growth of the transformed function, then to express this information in terms of the growth of $f$. An illustration of this approach rounds out the paper.

\section{Results}

The main result provides general estimates for the coefficients of the function (1).

Theorem 1. (cf. [6, pp.4406-08]) Suppose that $f$ is a transcendental entire function given by (1). Suppose also that $h(z)=\sum_{\mathbf{j} \geq \mathbf{0}} A_{\mathbf{j}} \mathbf{z}^{\mathbf{j}}$ is a fully indexed analytic function, with indexing set $\left\{\mathbf{r}_{\mathbf{N}}: \mathbf{N} \in(\mathbb{N} \cup\{0\})^{n}\right\}$, which is such that

$$
F(\mathbf{z})=\sum_{\mathbf{j} \geq \mathbf{0}} \frac{a_{\mathbf{j}}}{A_{\mathbf{j}}} \mathbf{z}^{\mathbf{j}}
$$

is entire. Then the inequalities

$$
\left|a_{\mathbf{j}}\right| \mathbf{r}^{\mathbf{j}} \leq \mu(\mathbf{r}, f) \frac{A_{\mathbf{j}}}{A_{\mathbf{N}}} \mathbf{r}_{\mathbf{N}}^{\mathbf{j}-\mathbf{N}}
$$

where $\mathbf{N}=\mathbf{N}(\mathbf{r}, f)$, hold for all $\mathbf{j} \geq \mathbf{0}$ and all $\mathbf{r}$ in the set

$$
\mathbf{S}=\left\{\operatorname{tr}_{\mathbf{N}(\mathbf{t}, F)}: \mathbf{t} \geq \mathbf{0}\right\} \text {. }
$$

The set $\mathbf{S}$ is such that, for all $\mathbf{r}_{2} \geq \mathbf{r}_{1} \geq 0$,

$$
\operatorname{logmeas}\left(\mathbf{S} \cap \Delta_{\mathbf{r}_{1}, \mathbf{r}_{2}}\right) \geq \operatorname{logmeas}\left(\Delta_{\mathbf{r}_{1}^{\prime}, \mathbf{r}_{2}^{\prime}}\right) \text {, }
$$

where $\mathbf{r}_{2}^{\prime} \geq \mathbf{r}_{1}^{\prime} \geq \mathbf{0}$ are any points such that, for all $\mathbf{t} \in \Delta_{\mathbf{r}_{1}^{\prime}, \mathbf{r}_{2}^{\prime}}$, we have $\mathbf{t} \boldsymbol{\rho}_{\mathbf{N}(\mathbf{t}, F)} \in$ $\Delta_{\mathbf{r}_{1}, \mathbf{r}_{2}}$.

The $\mathbf{r}_{1}$ and $\mathbf{r}_{2}$ in (11) are not be confused with the elements $\mathbf{r}_{\mathbf{N}}$ of the indexing set for $h$; the latter are always indicated by a boldface subscript.

Results that are more useful in applications can be obtained from Theorem 1 by making specific choices of $h$. More particularly, we will take $h=h_{\alpha}$, where $h_{\alpha}$ is given by (7), and make specific choices of $\alpha$, as in the following corollary.

Corollary 1. (cf. [6, Theorem 1]) Define $\alpha(t)$ by

$$
\alpha(0)=0, \quad \alpha^{\prime}(t)= \begin{cases}\epsilon /\left(t \log t \cdots\left(\log _{q+1} t\right)^{1+\epsilon}\right), & t \geq t_{0}, \\ \alpha^{\prime}\left(t_{0}\right), & 0 \leq t \leq t_{0},\end{cases}
$$

where $\epsilon$ is a positive number, $q$ is a non-negative integer, $\log _{q+1}$ is the $(q+1)$ th iterated logarithm, and $t_{0}$ is the $(q+1)$ th iterated exponential evaluated at 1 . Let $\alpha_{\mathbf{j}}$ and $\boldsymbol{\rho}_{\mathbf{j}}$ be given by (4) and (5), and let $\mathbf{S}$ be the set of Theorem 1 that corresponds to $h_{\alpha}$. Then for all $\mathbf{r}_{1} \geq 0$ and $\mathbf{r}_{2} \geq e^{1+\epsilon} \mathbf{r}_{1}$,

$$
\operatorname{logmeas}\left(\mathbf{S} \cap \Delta_{\mathbf{r}_{1}, \mathbf{r}_{2}}\right) \geq \operatorname{logmeas}\left(\Delta_{\mathbf{r}_{1}, e^{-1-\epsilon} \mathbf{r}_{2}}\right) \text {. }
$$

Remarks. (a) Values of $\mathbf{r} \geq \mathbf{0}$ at which the inequalities (9) hold are referred to here as normal for $f$ with respect to $h$; all other $\mathbf{r} \geq \mathbf{0}$ are exceptional. Since the right-hand side of (9) is less than 1 if $\mathbf{j} \neq \mathbf{N}$, the central index is unique at every normal value. 
(b) If $a_{\mathbf{0}}=f(\mathbf{0}) \neq 0$, then, taking $\mathbf{j}=\mathbf{0}$ in (9), we have

$$
\log \left(A_{\mathbf{N}} \mathbf{r}_{\mathbf{N}}^{\mathbf{N}}\right) \leq \log (\mu(\mathbf{r}, f))+O(1)
$$

as $\mathbf{r} \rightarrow \infty$ through normal values. This inequality can be used to estimate $\mathbf{N}$ in terms of $\mu$, cf. [10, pp 331-332].

(c) Suppose, on the other hand, that $a_{\mathbf{0}}=0$. Evidently all $\mathbf{r} \geq \mathbf{0}$ that are normal for $f+1$ are also normal for $f$, and further, if $\mathbf{r} \geq \mathbf{0}$ is such that $\mu(\mathbf{r}, f)>1$, then $\mu(\mathbf{r}, f)=\mu(\mathbf{r}, f+1)$ and $\mathbf{N}(\mathbf{r}, f)=\mathbf{N}(\mathbf{r}, f+1)$. Thus (14) holds in this case for all $\mathbf{r} \geq \mathbf{0}$ that are normal for $f+1$ and such that $\mu(\mathbf{r}, f)>1$.

Our second result, a so-called Clunie theorem, provides estimates for those terms of the function (1) that are reasonably far from the maximum term. To state the result we need some additional notation, cf. [7, formulas (6), (7) and (8)]. With $\mathbf{N}=\left(N_{1}, \ldots, N_{n}\right)$, we write

$$
N^{*}=\|\mathbf{N}\|=\max _{1 \leq j \leq n} N_{j}, \quad N_{*}=\min _{1 \leq j \leq n} N_{j},
$$

and define the following quantities:

$$
\lambda_{j}=\lambda_{j}(\beta)=\sqrt{\frac{60 \beta \log N^{*}}{\alpha^{\prime}\left(N_{j}\right)}}, \quad 1 \leq j \leq n,
$$

where $\alpha$ is given by (12) for some $\epsilon$ and $q$, and $\beta$ is a positive constant;

$$
\boldsymbol{\lambda}=\left(\lambda_{1}, \cdots, \lambda_{n}\right)
$$

and

$$
\mathbf{k}= \begin{cases}\boldsymbol{\lambda} & \text { if } \boldsymbol{\lambda} \leq \mathbf{N} \\ \boldsymbol{\lambda}^{2} / \mathbf{N} & \text { if } \boldsymbol{\lambda} \geq \mathbf{N}\end{cases}
$$

We have:

Theorem 2. (cf. [7, Theorem 2]) Suppose that $f$ is a transcendental entire function given by (1), that $\alpha$ is given by (12) for some $\epsilon$ and $q$, and that $\beta$ is a positive constant. Suppose that $\mathbf{R} \geq \mathbf{0}$ is normal for $f$ with respect to $h_{\alpha}$ given by (7), and let $\mathbf{r} \geq \mathbf{0}$ be such that

$$
\left|\log \frac{\mathbf{r}}{\mathbf{R}}\right| \leq \frac{1}{\mathbf{k}}
$$

where $\mathbf{k}$ is given by (17) with $\mathbf{N}=\mathbf{N}(\mathbf{R}, f)$. Then, given $\mathbf{p} \geq \mathbf{0}$, there are constants $C=C(\mathbf{p})$ and $N_{0}=N_{0}(\mathbf{p}, q, \epsilon, \beta)$ such that, if $N_{*} \geq N_{0}$, we have

$$
\sum_{\left|\mathbf{j}-\mathbf{N} \mathbf{1}_{\ell}\right| \geq \mathbf{k} \mathbf{1}_{\ell}} \mathbf{j}^{\mathbf{p}}\left|a_{\mathbf{j}}\right| \mathbf{r}^{\mathbf{j}} \leq C N^{* n-1-2 \beta} \mathbf{N}^{\mathbf{p}}\left|a_{\mathbf{N}}\right| \mathbf{r}^{\mathbf{N}}, \quad 1 \leq \ell \leq n .
$$

We prove also:

Theorem 3. (cf. [7, Theorem 3]) Suppose that $f$ is a transcendental entire function given by (1), that $\alpha$ is given by (12) for some $\epsilon$ and $q$, and that $\gamma$ is a positive constant. Suppose that $\mathbf{R} \geq \mathbf{0}$ is normal for $f$ with respect to $h_{\alpha}$ given by (7), and let $\mathbf{Z}$ be such that $|\mathbf{Z}|=\mathbf{R}$ and

$$
|f(\mathbf{Z})| \geq \eta M(\mathbf{R}, f)
$$


where $\eta$ depends on $\mathbf{N}=\mathbf{N}(\mathbf{R}, f)$ and satisfies $0<\eta \leq 1$ and

$$
\eta\|\mathbf{N}\|^{\gamma} \rightarrow \infty
$$

as $\mathbf{N} \rightarrow \infty$. Let $\mathbf{z}$ be such that

$$
\left|\log \frac{\mathbf{z}}{\mathbf{Z}}\right|=o\left(\eta \frac{1}{\mathbf{k}}\right)
$$

as $\mathbf{R} \rightarrow \infty$, where $\mathbf{k}$ is given by (17) with $\mathbf{N}=\mathbf{N}(\mathbf{R}, f)$. Suppose that $\mathbf{p} \geq \mathbf{0}$. If

$$
\log N^{*} \leq \frac{N_{*}^{2} \alpha^{\prime}\left(N_{*}\right)}{120\left(n-1+\|\mathbf{p}\|_{\circ}+\gamma\right)}
$$

then

$$
f^{(\mathbf{p})}(\mathbf{z})=\left(1+O\left(\eta^{-1} \sigma\right)+o(1)\right)\left(\frac{\mathbf{N}}{\mathbf{z}}\right)^{\mathbf{p}} f(\mathbf{z})
$$

as $\mathbf{R} \rightarrow \infty$, where

$$
|\sigma|=O\left(\sqrt{\frac{\log N^{*}}{N_{*}^{2} \alpha^{\prime}\left(N_{*}\right)}}\right) .
$$

Remarks. The effect of (23) is to ensure that $N_{*}$ is not too small compared with $N^{*}$. Evidently (see also the remark in [7, p. 216]), this hypothesis compromises the usefulness of Theorem 3, since one rarely possesses information of this kind about the central index. (The case $n=1$ is an exception, since then $N_{*}=N^{*}$ and (23) holds for all large z.) Nevertheless, for $n \geq 2$ no general result leading to the conclusion

$$
f^{(\mathbf{p})}(\mathbf{z})=(1+o(1))\left(\frac{\mathbf{N}}{\mathbf{z}}\right)^{\mathbf{p}} f(\mathbf{z})
$$

as $\mathbf{z} \rightarrow \infty$ through a reasonably large set is possible without some such hypothesis as $(23)$.

To see this, consider the example

$$
E(\mathbf{z})=e^{z_{1}}+\cdots+e^{z_{n}} .
$$

Given any $\mathbf{r} \geq \mathbf{0}$, the largest term in the power series for $E$ when $|\mathbf{z}|=\mathbf{r}$ is $\mu\left(r_{j}, e^{z}\right)$, where $j$ is any subscript for which $r_{j}=\|\mathbf{r}\|$. With the same $j$, a value of the central index is $N\left(r_{j}\right) \mathbf{1}_{j}$, where $N$ is the central index of $e^{z}$. More generally, the central index of $E, \mathbf{N}(\mathbf{r}, E)$, is any nonzero member of the set

$$
\left\{\left[\frac{r_{j}}{\|\mathbf{r}\|}\right] N(\|\mathbf{r}\|) \mathbf{1}_{j}: 1 \leq j \leq n\right\}
$$

where [ ] denotes the integer part. Thus (23) fails for $E$ for all large z, except those for which $|\mathbf{z}|=r \mathbf{1}$ for some $r>0$. Values $r \mathbf{1}$ are not normal, however, since $\mathbf{N}$ is not unique there (cf. Remark (a) following Theorem 1 Corollary 1). So Theorem 3 gives no information about the partial derivatives of $E$. But this is not surprising, since (26) is actually false for all large $\mathbf{z}$ for which it makes sense, that is, for all large $\mathbf{z}$ for which the central index is uniquely defined. For if (26) were to hold at $\mathbf{z}$, we would have, taking $\mathbf{p}=\mathbf{1}_{j}$,

$$
e^{z_{j}}=(1+o(1))\left[\frac{\left|z_{j}\right|}{\|\mathbf{z}\|}\right] \frac{N(\|\mathbf{z}\|)}{z_{j}}\left(e^{z_{1}}+\cdots+e^{z_{n}}\right) .
$$


This is impossible unless $\left|z_{j}\right|=\|\mathbf{z}\|$ for all $j$, and in that case the central index is not uniquely defined.

Theorem 3 is thus effectively useless for the investigation of partial differential equations. The next theorem, a two-variable version of which can be assembled from comments in [8, p. 573-75], provides a way of analysing partial differential equations using Wiman-Valiron methods. An illustration of its use, involving the same function $E$ that we have just considered, is given in the last section of the paper.

Theorem 4. Let $\Phi: \mathbb{C}^{n} \rightarrow \mathbb{C}^{n}$ be defined by

$$
\mathrm{z}=\Phi(\zeta)=\zeta^{1} \zeta
$$

Then the following hold.

(a) $\boldsymbol{\Phi}$ maps $\mathbb{C}$ onto $\mathbb{C}$ and maps $\{\boldsymbol{\zeta}: \boldsymbol{\zeta}>\mathbf{0}\}$ one-one onto $\{\mathbf{z}: \mathbf{z}>\mathbf{0}\}$. The Jacobian of the transformation $\log \zeta \rightarrow \log \mathbf{z}$ is

$$
\frac{\partial(\log \mathbf{z})}{\partial(\log \zeta)}=n+1
$$

(b) For all $\mathbf{j}_{\mathbf{1}}$ and $\mathbf{j}_{\mathbf{2}}$, if $\operatorname{deg}\left(\boldsymbol{\Phi}(\boldsymbol{\zeta})^{\mathbf{j}_{1}}\right)=\operatorname{deg}\left(\boldsymbol{\Phi}(\boldsymbol{\zeta})^{\mathbf{j}_{2}}\right)$ then $\mathbf{j}_{\mathbf{1}}=\mathbf{j}_{\mathbf{2}}$.

(c) With $f$ given by (1) and

$$
\mathbf{N}(\boldsymbol{\zeta}, \mathcal{F})=\mathbf{N}(\mathbf{z}, f)+\|\mathbf{N}(\mathbf{z}, f)\|_{\circ} \mathbf{1}
$$

(d) Suppose that $\alpha$ is given by (12) for some $\epsilon$ and $q$, and that $\gamma$ is a positive number. Suppose that $\mathcal{R}>\mathbf{0}$ is normal for $\mathcal{F}$ with respect to $h_{\alpha}$ given by (7), and let $\mathcal{Z}$ be such that $|\mathcal{Z}|=\mathcal{R}$ and

$$
|\mathcal{F}(\mathcal{Z})| \geq \eta M(\mathcal{R}, \mathcal{F})
$$

where $\eta$ depends on $\mathcal{N}=\mathbf{N}(\mathcal{R}, \mathcal{F})$ and satisfies $0<\eta \leq 1$ and

$$
\eta\|\mathcal{N}\|^{\gamma} \rightarrow \infty
$$

as $\mathcal{N} \rightarrow \infty$. Let $\boldsymbol{\zeta}$ be such that

$$
\left|\log \frac{\zeta}{\mathcal{Z}}\right|=o\left(\eta \frac{1}{\mathbf{k}}\right)
$$

as $\boldsymbol{\mathcal { R }} \rightarrow \infty$, where $\boldsymbol{k}$ is given by (17) with $\mathbf{N}=\mathcal{N}$. If $\mathbf{p} \geq \mathbf{0}$, then

$$
\mathcal{F}^{(\mathbf{p})}(\boldsymbol{\zeta})=\left(1+O\left(\eta^{-1} \sigma\right)+o(1)\right)\left(\frac{\mathcal{N}}{\boldsymbol{\zeta}}\right)^{\mathbf{p}} \mathcal{F}(\boldsymbol{\zeta})
$$

as $\mathcal{R} \rightarrow \infty$, where

$$
|\sigma|=O\left(\sqrt{\frac{\log \|\mathcal{N}\|}{\|\mathcal{N}\|^{2} \alpha^{\prime}(\|\mathcal{N}\|)}}\right) .
$$


Remark. Equation (36) is most useful when $\eta^{-1} \sigma=o(1)$ as $\mathcal{R} \rightarrow \infty$. In view of (37) and (12), we have

$$
\sigma \leq \epsilon^{-1 / 2}\|\mathcal{N}\|^{-1 / 2} \log \|\mathcal{N}\|\left(\log _{2}\|\mathcal{N}\| \cdots\left(\log _{q+1}\|\mathcal{N}\|\right)^{1+\epsilon}\right)^{1 / 2}
$$

The smallest allowable $\eta$ from which a useful conclusion can be drawn from Theorem 4 is thus

$$
\eta_{0}(\mathcal{N})=\|\mathcal{N}\|^{-1 / 2} \log \|\mathcal{N}\|\left(\log _{2}\|\mathcal{N}\| \cdots\left(\log _{q+1}\|\mathcal{N}\|\right)^{1+\epsilon^{\prime}}\right)^{1 / 2}
$$

where $\epsilon^{\prime}>\epsilon$. The corresponding $\gamma$ is $\gamma=1 / 2$. We thus have:

Corollary 1. Suppose that $\alpha$ is given by (12) for some $\epsilon$ and $q$, and that $\mathcal{R}>\mathbf{0}$ is normal for $\mathcal{F}$ with respect to $h_{\alpha}$ given by $(7)$. Let $\mathcal{Z}$ be such that $|\mathcal{Z}|=\mathcal{R}$ and

$$
|\mathcal{F}(\mathcal{Z})| \geq \eta M(\mathcal{R}, \mathcal{F})
$$

where $\eta$ satisfies $\eta_{0}(\mathcal{N})<\eta \leq 1$ and $\mathcal{N}=\mathbf{N}(\mathcal{R}, \mathcal{F})$. Let $\boldsymbol{\zeta}$ be such that

$$
\left|\log \frac{\zeta}{\mathcal{Z}}\right|=o\left(\eta \frac{1}{\mathbf{k}}\right)
$$

as $\mathcal{R} \rightarrow \infty$, where $\boldsymbol{k}$ is given by (17) with $\mathbf{N}=\mathcal{N}$. If $\mathbf{p} \geq \mathbf{0}$ then

$$
\mathcal{F}^{(\mathbf{p})}(\boldsymbol{\zeta})=(1+o(1))\left(\frac{\mathcal{N}}{\boldsymbol{\zeta}}\right)^{\mathbf{p}} \mathcal{F}(\boldsymbol{\zeta})
$$

as $\mathcal{R} \rightarrow \infty$.

Remark. When $\eta=1$ in Theorem 2 Corollary 1, condition (40) is equivalent to

$$
|\log \boldsymbol{\zeta} / \mathcal{Z}|=o\left(\|\mathcal{N}\|^{-1 / 2}(\log \|\mathcal{N}\|)^{-1}\left(\log _{2}\|\mathcal{N}\| \cdots\left(\log _{q+1}\|\mathcal{N}\|\right)^{1+\epsilon}\right)^{-1 / 2}\right) .
$$

In the case $n=1$ this is the condition implicit in [[10]]. A recent result of Bergweiler [1, Theorem 1.1 and Remark 1], proved using different methods, shows that in the case $n=1$ it is possible to replace this condition by

$$
|\log \zeta / \mathcal{Z}|=o\left(\|\mathcal{N}\| \log \|\mathcal{N}\| \log _{2}\|\mathcal{N}\| \cdots\left(\log _{q+1}\|\mathcal{N}\|\right)^{1+\epsilon}\right)^{-1 / 2}
$$

and that this is sharp. (In fact he shows slightly more.) This suggests that the numbers $\lambda_{j}$ in (16) are too large by a factor of $\left(\log N^{*}\right)^{1 / 2}$. While this has little or no practical consequence, it is interesting evidence of a small gap in the methods used here.

\section{The central index}

When $n=1$ the central index behaves in a particularly simple way: we have $\mathbf{N}\left(\mathbf{r}_{1}, f\right) \leq \mathbf{N}\left(\mathbf{r}_{2}, f\right)$ for $\mathbf{0} \leq \mathbf{r}_{1} \leq \mathbf{r}_{2}$. But the behaviour may be more complicated for $n \geq 2$. For $n=2$, for example, the central index of

$$
\sum_{j=0}^{\infty} \frac{1}{j !}\left(z_{1}^{j}+z_{1}^{j-1} z_{2}+\cdots+z_{1} z_{2}^{j-1}+z_{2}^{j}\right)
$$

has the form $(N, 0)$ if $\left|z_{1}\right|>\left|z_{2}\right|$ and $(0, N)$ if $\left|z_{2}\right|>\left|z_{1}\right|$. Thus, by increasing $|\mathbf{z}|$ we do not necessarily increase $\mathbf{N}$.

We consider here some general properties of the central index that are useful in what follows, beginning with a several-variables version of the Newton polygon [13, p. 28 et seq.]. 
With $f$ given by (1), let

$$
\mathbf{B}=\left\{\left(\mathbf{j},-\log \left|a_{\mathbf{j}}\right|\right): \mathbf{j} \geq \mathbf{0} \text { and } a_{\mathbf{j}} \neq 0\right\},
$$

a set in $\mathbb{R}^{n+1}$, and consider the relation of $\mathbf{B}$ to the hyperplane $\mathbf{H}_{\mathbf{r}, b}$ in $\mathbb{R}^{n+1}$ defined by

$$
\mathbf{x} \cdot(\log \mathbf{r},-1)=b
$$

where $\mathbf{r}>\mathbf{0}$ and $b \in \mathbb{R}$ are fixed. Let us write

$$
\mathbf{H}_{\mathbf{r}, b}^{+}=\left\{\mathbf{x} \in \mathbb{R}^{n+1}: \mathbf{x} \cdot(\log \mathbf{r},-1)>b\right\} .
$$

Since $f$ is entire, we have $\log \left|a_{\mathbf{j}}\right| /\|\mathbf{j}\| \rightarrow-\infty$ as $\mathbf{j} \rightarrow \infty$, and thus

$$
\left(\mathbf{j},-\log \left|a_{\mathbf{j}}\right|\right) \cdot(\log \mathbf{r},-1)=\mathbf{j} \cdot \log \mathbf{r}+\log \left|a_{\mathbf{j}}\right| \rightarrow-\infty
$$

as $\mathbf{j} \rightarrow \infty$, for each fixed $\mathbf{r}>\mathbf{0}$. It follows that $\mathbf{B} \cap \mathbf{H}_{\mathbf{r}, b}^{+}=\emptyset$ if $b$ is large enough. There is thus a smallest value of $b$ for which $\mathbf{H}_{\mathbf{r}, b}$ supports $\mathbf{B}$, in the sense that $\mathbf{B} \cap \mathbf{H}_{\mathbf{r}, b}^{+}=\emptyset$ and $\mathbf{B} \cap \mathbf{H}_{\mathbf{r}, b} \neq \emptyset$ (and is necessarily finite, from (42)). Let us denote this value of $b$ by $b(\mathbf{r})$.

Suppose that $\left(\mathbf{N},-\log \left|a_{\mathbf{N}}\right|\right) \in \mathbf{B} \cap \mathbf{H}_{\mathbf{r}, b(\mathbf{r})}$. Then, for all $\mathbf{j}$,

$$
\mathbf{j} \cdot \log \mathbf{r}+\log \left|a_{\mathbf{j}}\right| \leq b(\mathbf{r})=\mathbf{N} \cdot \log \mathbf{r}+\log \left|a_{\mathbf{N}}\right|,
$$

that is

$$
\log \left(\left|a_{\mathbf{j}} / a_{\mathbf{N}}\right| \mathbf{r}^{\mathbf{j}-\mathbf{N}}\right) \leq 0
$$

so that $\mathbf{N}$ is a central index on $f$. Note also that, from $(43), b(\mathbf{r})=\log \mu(\mathbf{r}, f)$. Conversely, if $\mathbf{N}=\mathbf{N}(\mathbf{r}, f)$ for some $\mathbf{r}>\mathbf{0}$, and $b$ is such that $\mathbf{H}_{\mathbf{r}, b}$ supports $\mathbf{B}$, then $\left(\mathbf{N},-\log \left|a_{\mathbf{N}}\right|\right) \in \mathbf{B} \cap \mathbf{H}_{\mathbf{r}, b}$. The central index can thus be characterized as follows: $\mathbf{N}=\mathbf{N}(\mathbf{r}, f)$ for some $\mathbf{r}>\mathbf{0}$ if and only if the hyperplane $\mathbf{H}_{\mathbf{r}, b}$ that supports $\mathbf{B}$ contains $\left(\mathbf{N},-\log \left|a_{\mathbf{N}}\right|\right)$. Expressed slightly differently, $\mathbf{r}>\mathbf{0}$ is a point for which the central index has a given value $\mathbf{N}$ if and only if $\mathbf{B}$ is supported by the hyperplane through $\left(\mathbf{N},-\log \left|a_{\mathbf{N}}\right|\right)$ with normal $(\log \mathbf{r},-1)$.

This approach can be extended to cover values of $\mathbf{r} \geq \mathbf{0}$, and for this purpose we define $\log 0=-\infty$ and $0 \cdot( \pm \infty)=0$. Then $\mathbf{H}_{\mathbf{r}, b}$ is defined for all $\mathbf{r} \geq \mathbf{0}$, with the exception of those $\mathbf{r}$ for which all terms in the series for $f(\mathbf{r})$ vanish. $\left(\mathbf{H}_{\mathbf{r}, b}\right.$ is no longer a hyperplane if one or more of the components of $\mathbf{r}$ is 0 but this is of no consequence.) It follows as before from (42) - which remains true, although the left-hand side may be $-\infty$ for certain $\mathbf{j}$ - that there is a smallest value of $b$ for which $\mathbf{H}_{\mathbf{r}, b}$ supports $\mathbf{B}$, in the sense that $\mathbf{B} \cap \mathbf{H}_{\mathbf{r}, b}^{+}=\emptyset$ and $\mathbf{B} \cap \mathbf{H}_{\mathbf{r}, b}$ is non-empty (and finite). All points of $\mathbf{B} \cap \mathbf{H}_{\mathbf{r}, b}$ correspond to central indices, and the previous characterization of the central index carries over.

The set $\mathfrak{N}=\cap_{\mathbf{r}>\mathbf{0}} \operatorname{comp}\left(\mathbf{H}_{\mathbf{r}, b(\mathbf{r})}^{+}\right)$, where 'comp' denotes complement, is the Newton polytope of $f$; it is the closed, convex hull of $\mathbf{B}$. At the risk of repetition: if $\mathbf{r} \geq \mathbf{0}$ is such that not all terms of $f(\mathbf{r})$ are 0 , and if $\mathbf{H}_{\mathbf{r}, b}$ supports $\mathfrak{N}$, then $\mathbf{H}_{\mathbf{r}, b} \cap \mathbf{B} \neq \emptyset$ and is finite. All points of $\mathbf{H}_{\mathbf{r}, b} \cap \mathbf{B}$ then correspond to central indices of $f$ at $\mathbf{r}$, and $b=\log \mu(\mathbf{r}, f)$.

Let us write

$$
\mathbf{S}_{\mathbf{N}}=\mathbf{S}_{\mathbf{N}}(f)=\{\log \mathbf{r}: \mathbf{r}>\mathbf{0} \text { and } \mathbf{N}(\mathbf{r}, f)=\mathbf{N}\} .
$$

Evidently $\log \mathbf{r} \in \mathbf{S}_{\mathbf{N}}$ if and only if (44) holds for all $\mathbf{j}$. Denoting the interior of $\mathbf{S}_{\mathbf{N}}$ by int $\mathbf{S}_{\mathbf{N}}$, we have: 
Lemma 5. (a) The sets $\mathbf{S}_{\mathbf{N}}$ are closed and convex.

(b) If $\mathbf{N}_{1} \neq \mathbf{N}_{2}$ then $\mathbf{S}_{\mathbf{N}_{1}} \cap \operatorname{int} \mathbf{S}_{\mathbf{N}_{2}}=\emptyset$.

(c) For any $r>0$, the set

$$
\{\log \mathbf{r}: \mathbf{0}<\mathbf{r}<r \mathbf{1}\}
$$

is intersected by only finitely many $\mathbf{S}_{\mathbf{N}}$.

(d) The sets $\mathbf{S}_{\mathbf{N}}$ that have non-empty interior tessellate $\mathbb{R}^{n}$.

Proof. Part (a) follows from the observation following (45), since if $\mathbf{r}_{1}, \mathbf{r}_{2} \in \mathbf{S}_{\mathbf{N}}$ and $0 \leq \theta \leq 1$, then from (44),

$$
\log \left(\left|a_{\mathbf{j}} / a_{\mathbf{N}}\right|\left(\mathbf{r}_{1}^{\theta} \mathbf{r}_{2}^{1-\theta}\right)^{\mathbf{j}-\mathbf{N}}\right)=\theta \log \left(\left|a_{\mathbf{j}} / a_{\mathbf{N}}\right| \mathbf{r}_{1}^{\mathbf{j}-\mathbf{N}}\right)+(1-\theta) \log \left(\left|a_{\mathbf{j}} / a_{\mathbf{N}}\right| \mathbf{r}_{2}^{\mathbf{j}-\mathbf{N}}\right) \leq 0 .
$$

For (b), suppose that $\log \mathbf{r}_{0} \in \mathbf{S}_{\mathbf{N}_{1}} \cap \operatorname{int} \mathbf{S}_{\mathbf{N}_{2}}$ for some $\mathbf{N}_{1} \neq \mathbf{N}_{2}$, and suppose that $\mathbf{N}_{1}$ differs from $\mathbf{N}_{2}$ in the $j$ th component. Since, necessarily, $\mathbf{r}_{0}>0$, we can find $\epsilon>0$ sufficiently small that, with $\mathbf{r}=\mathbf{r}_{0} \pm \epsilon \mathbf{1}_{j}$, we have $\mathbf{r}>\mathbf{0}$ and $\log \mathbf{r} \in \operatorname{int} \mathbf{S}_{\mathbf{N}_{2}}$. Since

$$
\left|a_{\mathbf{N}_{1}}\right| \mathbf{r}_{0} \mathbf{N}=\left|a_{\mathbf{N}_{2}}\right| \mathbf{r}_{0} \mathbf{N}^{2}
$$

we have

$$
\left|a_{\mathbf{N}_{1}}\right| \mathbf{r}^{\mathbf{N}_{1}}>\left|a_{\mathbf{N}_{2}}\right| \mathbf{r}^{\mathbf{N}_{2}}
$$

for some choice of the \pm sign. Thus $\mathbf{N}_{2} \neq \mathbf{N}(\mathbf{r}, f)$, a contradiction.

For (c), note first that the set

$$
\{\mathbf{N}(\mathbf{r}, f): \mathbf{0}<\mathbf{r}<r \mathbf{1}\}
$$

is finite for all $r>0$. For otherwise there is an $r>0$, and there are points $\mathbf{0}<\mathbf{r}_{k}<$ $r \mathbf{1}$, such that, with $\mathbf{N}_{k}=\mathbf{N}\left(\mathbf{r}_{k}, f\right)$, we have $\mathbf{N}_{k} \rightarrow \infty$ as $k \rightarrow \infty$. Write $\mathbf{N}_{k}=$ $\left(N_{1}(k), \ldots, N_{n}(k)\right)$. Since

$$
\left|a_{\mathbf{N}_{k}}\right| \mathbf{r}_{k}^{\mathbf{N}_{k}-\mathbf{N}_{1}} \geq\left|a_{\mathbf{N}_{1}}\right|>0,
$$

and since the left-hand side of (47) tends to 0 as $k \rightarrow \infty$ if $\mathbf{N}_{k} \geq \mathbf{N}_{1}$, we deduce that $N_{j}=\liminf _{k \rightarrow \infty} N_{j}(k)<N_{j}(1)$ for some $1 \leq j \leq n$. We may assume without loss of generality that $j=1$ and, by taking a subsequence if necessary, that $N_{1}(k)=N_{1}$ for all $k$. We repeat the argument and deduce that $N_{j}=\liminf _{k \rightarrow \infty} N_{j}(k)<N_{j}(1)$ for some $2 \leq j \leq n$, and so on. The upshot is that we can find a subsequence of $\mathbf{N}_{k}$ on which every component is bounded, a contradiction. Thus the set (46) is finite, and (c) follows.

We deduce from (c) that, for any $r>0$,

$$
\{\log \mathbf{r}: \mathbf{0}<\mathbf{r}<r \mathbf{1}\} \backslash \cup_{\mathbf{N}} \overline{\operatorname{int} \mathbf{S}_{\mathbf{N}}}
$$

is open. Also, since from (a) each $\mathbf{S}_{\mathbf{N}}$ is closed and convex, either $\operatorname{int} \mathbf{S}_{\mathbf{N}}=\emptyset$ or $\mathbf{S}_{\mathbf{N}}=\overline{\operatorname{int} \mathbf{S}_{\mathbf{N}}}$. It follows from (c) that the set (48) is contained in a finite union of those $\mathbf{S}_{\mathbf{N}}$ that have empty interior. It is therefore empty, which proves (d) and completes the proof of the lemma.

Remarks. (a) The central index is uniquely defined precisely on the set of $\mathbf{r}$ for which $\log \mathbf{r} \in \cup_{\mathbf{N}}$ int $\mathbf{S}_{\mathbf{N}}$. (For such $\mathbf{r}, H_{\mathbf{r}, b(\mathbf{r})}$ meets $\mathfrak{N}$ at a single point.) For certainly the central index is uniquely defined on this set, from Lemma 6 (b). On the other hand, if $\mathbf{r}$ is not in the set then, from Lemma 6 (d), $\log \mathbf{r}$ is a boundary point of (one and therefore) at least two sets $\mathbf{S}_{\mathbf{N}_{1}}$ and $\mathbf{S}_{\mathbf{N}_{2}}, \mathbf{N}_{1} \neq \mathbf{N}_{2}$, that have non-empty 
interiors. Thus $\mathbf{r} \in \mathbf{S}_{\mathbf{N}_{1}} \cap \mathbf{S}_{\mathbf{N}_{2}}$ and $\mathbf{N}_{1}$ and $\mathbf{N}_{2}$ are both values of the central index at $\mathbf{r}$.

(b) Suppose that $\mathbf{r}$ and $\mathbf{s}$ are fixed, with $\mathbf{r}>\mathbf{0}$, and that $t \in \mathbb{R}$. If, for some $\mathbf{N}$, we have $\log \mathbf{r}+t \mathbf{s} \in \mathbf{S}_{\mathbf{N}}$ for all $t$ in a certain interval, then

$$
\frac{d}{d t} \log \mu\left(\mathbf{r e}^{t \mathbf{s}}, f\right)=\frac{d}{d t}\left(\log \left(\left|a_{\mathbf{N}}\right| \mathbf{r}^{\mathbf{N}}\right)+t \mathbf{s} \cdot \mathbf{N}\right)=\mathbf{s} \cdot \mathbf{N}
$$

Thus, for all $\mathbf{R}_{1}, \mathbf{R}_{2}>0$ we have, taking $\mathbf{r}=\mathbf{R}_{1}$ and $\mathbf{s}=\log \left(\mathbf{R}_{2} / \mathbf{R}_{1}\right)$,

$$
\log \mu\left(\mathbf{R}_{2}, f\right)-\log \mu\left(\mathbf{R}_{1}, f\right)=\log \left(\mathbf{R}_{2} / \mathbf{R}_{1}\right) \cdot \int_{0}^{1} \mathbf{N}\left(\mathbf{R}_{1} \mathbf{e}^{t \log \left(\mathbf{R}_{2} / \mathbf{R}_{1}\right)}, f\right) d t .
$$

We deduce from (50) that if $\mathbf{R}_{2} \geq \mathbf{R}_{1}>\mathbf{0}$ then

$$
\mathbf{N}\left(\mathbf{R}_{1}, f\right) \cdot \log \left(\mathbf{R}_{2} / \mathbf{R}_{1}\right) \leq \log \mu\left(\mathbf{R}_{2}, f\right)-\log \mu\left(\mathbf{R}_{1}, f\right) \leq \mathbf{N}\left(\mathbf{R}_{2}, f\right) \cdot \log \left(\mathbf{R}_{2} / \mathbf{R}_{1}\right) .
$$

(c) Suppose that $\mathbf{s} \geq \mathbf{0}$ in (49). If, for some $\mathbf{N}$, we have $\log \mathbf{r}+t \mathbf{s} \in \mathbf{S}_{\mathbf{N}}$ for all $t$ in a certain interval, then $d^{2} / d t^{2}\left(\log \mu\left(\mathbf{r e}^{t \mathbf{s}}, f\right)=0\right.$, from (49). Also, if $t_{0}$ is such that $\log \mathbf{r}+t_{0} \mathbf{s} \in \mathbf{S}_{\mathbf{N} 1} \cap \mathbf{S}_{\mathbf{N} 2}$, where $\mathbf{N}_{1} \neq \mathbf{N}_{2}$, and if $\log \mathbf{r}+t \mathbf{s} \in \mathbf{S}_{\mathbf{N} 1}$ for all $t$ for which $t<t_{0}$ and $t$ is sufficiently close to $t_{0}$, while $\log \mathbf{r}+t \mathbf{s} \in \mathbf{S}_{\mathbf{N}_{2}}$ for all $t$ for which $t>t_{0}$ and $t$ is sufficiently close to $t_{0}$, then $\mathbf{s} \cdot \mathbf{N}_{1} \leq \mathbf{s} \cdot \mathbf{N}_{2}$. For otherwise we would have, with $\mathbf{r}_{0}=\mathbf{r e}^{t_{0} \mathbf{s}}$,

$$
\left|a_{\mathbf{N}_{1}}\right|\left(\mathbf{r}_{0} \mathbf{e}^{\left(t-t_{0}\right) \mathbf{s}}\right)^{\mathbf{N}_{1}}>\left|a_{\mathbf{N}_{2}}\right|\left(\mathbf{r}_{0} \mathbf{e}^{\left(t-t_{0}\right) \mathbf{s}}\right)^{\mathbf{N}_{2}}
$$

for all $t$ just greater than $t_{0}$, a contradiction. We deduce that if $\mathbf{s} \geq \mathbf{0}$ then $\log \mu\left(\mathbf{r e}^{t \mathbf{s}}, f\right)$ is a convex, non-decreasing function of $t$.

\section{Proof of Theorem 1}

We distinguish two cases. Suppose first that $\mathbf{t} \geq \mathbf{0}$ and that $\mu(\mathbf{t}, F)=0$. Then $\mathbf{N}(\mathbf{t}, F)$ is entirely ambiguous and can be any value. Nevertheless, since all terms of $f(\mathbf{t})$ are also necessarily zero, as are all terms of $f\left(\mathbf{t r}_{\mathbf{N}(\mathbf{t}, F)}\right)$, no matter what value we assign to $\mathbf{N}(\mathbf{t}, F)$, the inequalities (9) hold at $\mathbf{r}=\mathbf{t r}_{\mathbf{N}(\mathbf{t}, F)}$.

Suppose next that $\mathbf{t} \geq \mathbf{0}$ and that $\mu(\mathbf{t}, F)>0$. Let $\mathbf{N}=\mathbf{N}(\mathbf{t}, F)$, allowing the possibility that $\mathbf{N}(\mathbf{t}, F)$ may take more than one value. Then, for all $\mathbf{n}$,

$$
\frac{\left|a_{\mathbf{n}}\right|}{A_{\mathbf{n}}} \mathbf{t}^{\mathbf{n}} \leq \frac{\left|a_{\mathbf{N}}\right|}{A_{\mathbf{N}}} \mathbf{t}^{\mathbf{N}}
$$

Multiplying both sides of this inequality by $\mathbf{r}_{\mathbf{N}}^{\mathbf{n}-\mathbf{N}}$, we obtain

$$
\frac{\left|a_{\mathbf{n}}\right|\left(\operatorname{tr}_{\mathbf{N}}\right)^{\mathbf{n}}}{\left|a_{\mathbf{N}}\right|\left(\operatorname{tr}_{\mathbf{N}}\right)^{\mathbf{N}}} \leq \frac{A_{\mathbf{n}} \mathbf{r}_{\mathbf{N}}^{\mathbf{n}}}{A_{\mathbf{N}} \mathbf{r}_{\mathbf{N}}^{\mathbf{N}}}
$$

The right-hand side of (51) is less than 1 for $\mathbf{n} \neq \mathbf{N}$, from (6). We deduce that $\mathbf{N}=\mathbf{N}\left(\mathbf{t r}_{\mathbf{N}}, f\right)$ and that the inequalities (9) hold for $\mathbf{r}=\mathbf{t r}_{\mathbf{N}}$. This proves the first part of Theorem 1. (Let us note, incidentally, that we have shown that $\mathbf{N}(\mathbf{r}, f)$ is uniquely defined at all $\mathbf{r}$ of the form $\mathbf{r}=\operatorname{tr}_{\mathbf{N}(\mathbf{t}, F)}, \mathbf{t} \geq \mathbf{0}$, for which $\mu(\mathbf{r}, f)>0$.)

Concerning (11), suppose that $\mathbf{r}_{1}, \mathbf{r}_{2}, \mathbf{r}_{1}^{\prime}, \mathbf{r}_{2}^{\prime}$ are as in the statement of the Theorem 1. From Lemma 1 (d), we have

$$
\log \Delta_{\mathbf{r}_{1}^{\prime}, \mathbf{r}_{2}^{\prime}}=\cup_{\mathbf{N}}^{\#}\left(\log \Delta_{\mathbf{r}_{1}^{\prime}, \mathbf{r}_{2}^{\prime}}\right) \cap \mathbf{S}_{\mathbf{N}}(F),
$$


the ${ }^{\#}$ indicating that the union is taken over only those $\mathbf{S}_{\mathbf{N}}(F)$ that have non-empty interior. Thus, using the fact that the boundary of a convex set has measure zero,

$$
\begin{aligned}
\operatorname{logmeas} \Delta_{\mathbf{r}_{1}^{\prime}, \mathbf{r}_{2}^{\prime}} & =\sum_{\mathbf{N}}^{\#} \text { meas }\left(\left(\log \Delta_{\mathbf{r}_{1}^{\prime}, \mathbf{r}_{2}^{\prime}}\right) \cap \mathbf{S}_{\mathbf{N}}(F)\right) \\
& =\sum_{\mathbf{N}}^{\#} \operatorname{meas}\left(\log \mathbf{r}_{\mathbf{N}}+\left(\log \Delta_{\mathbf{r}_{1}^{\prime}, \mathbf{r}_{2}^{\prime}}\right) \cap \mathbf{S}_{\mathbf{N}}(F)\right) \\
& =\sum_{\mathbf{N}}^{\#} \operatorname{meas}\left(\log \mathbf{r}_{\mathbf{N}}+\left(\log \Delta_{\mathbf{r}_{1}^{\prime}, \mathbf{r}_{2}^{\prime}}\right) \cap \operatorname{int} \mathbf{S}_{\mathbf{N}}(F)\right) \\
& =\sum_{\mathbf{N}}^{\#} \operatorname{meas}\left(\left\{\log \left(\mathbf{t r}_{\mathbf{N}}(\mathbf{t}, F): \log \mathbf{t} \in\left(\log \Delta_{\mathbf{r}_{1}^{\prime}, \mathbf{r}_{2}^{\prime}}\right) \cap \operatorname{int} \mathbf{S}_{\mathbf{N}}(F)\right\}\right)\right. \\
& \leq \sum_{\mathbf{N}}^{\#} \operatorname{meas}\left(\left\{\log \left(\operatorname{tr}_{\mathbf{N}}(\mathbf{t}, F): \mathbf{t} \in \Delta_{\mathbf{r}_{1}^{\prime}, \mathbf{r}_{2}^{\prime}}\right\}\right)\right. \\
& =\operatorname{logmeas}\left(\left\{\mathbf{t r}_{\mathbf{N}(\mathbf{t}, F)}: \mathbf{t} \in \Delta_{\mathbf{r}_{1}^{\prime}, \mathbf{r}_{2}^{\prime}}\right\}\right) \\
& \leq \operatorname{logmeas}\left(\Delta_{\mathbf{r}_{1}, \mathbf{r}_{2}} \cap\left\{\mathbf{t r}_{\mathbf{N}(\mathbf{t}, F)}: \mathbf{t}>\mathbf{0}\right\}\right) \\
& =\operatorname{logmeas}\left(\Delta_{\mathbf{r}_{1}, \mathbf{r}_{2}} \cap \mathbf{S}\right),
\end{aligned}
$$

which completes the proof of Theorem 1.

Concerning Corollary 1 , let us note that

$$
\alpha(t)= \begin{cases}t \alpha^{\prime}\left(t_{0}\right), & 0 \leq t \leq t_{0} \\ t_{0} \alpha^{\prime}\left(t_{0}\right)+1-\left(\log _{q+1} t\right)^{-\epsilon}, & t \geq t_{0}\end{cases}
$$

so that, for all $j \geq 0$,

$$
\int_{0}^{j} \alpha(t) d t \leq K j
$$

where $K=\alpha^{\prime}\left(t_{0}\right)+1$. It follows that $\alpha_{j}$ given by (4) satisfies $\alpha_{j} \geq e^{-K j}$ for all $j$, and therefore

$$
\left|\frac{a_{\mathbf{j}}}{\alpha_{\mathbf{j}}} \mathbf{z}^{\mathbf{j}}\right| \leq\left|a_{\mathbf{j}}\left(e^{K} \mathbf{z}\right)^{\mathbf{j}}\right|
$$

for all $\mathbf{j}$. So $F$ given by (8) is entire. Further, with (5) in view we have, using the fact that $\alpha$ and $\alpha^{\prime}$ are non-negative,

$$
\mathbf{1} \leq \boldsymbol{\rho}_{\mathbf{n}} \leq e^{\int_{0}^{\infty} \alpha^{\prime}(t) d t} \mathbf{1}=e^{1+t_{0} \alpha^{\prime}\left(t_{0}\right)} \mathbf{1} \leq e^{1+\epsilon} \mathbf{1}
$$

for all $\mathbf{n}$. We may therefore apply Theorem 1 with $\mathbf{r}_{\mathbf{1}}^{\prime}=\mathbf{r}_{\mathbf{1}}$ and $\mathbf{r}_{\mathbf{2}}^{\prime}=e^{-1-\epsilon} \mathbf{r}_{\mathbf{2}}$, and Corollary 1 follows.

Remark. Taking $\mathbf{r}_{1}=\mathbf{1}$ and, for any $R \geq e^{1+\epsilon}, \mathbf{r}_{\mathbf{2}}=R \mathbf{1}$, we conclude that the set $\mathbf{S}$ in Theorem 1 Corollary 1 is such that

$$
\begin{aligned}
\operatorname{logmeas}\left(\mathbf{S} \cap \Delta_{\mathbf{1}, R \mathbf{1}}\right) & \geq(\log R-1-\epsilon)^{n} \\
& = \begin{cases}\log R-1-\epsilon, & n=1, \\
\left.(\log R)^{n}-n(1+\epsilon)(\log R)^{n-1}+O\left((\log R)^{n-2}\right)\right), & n \geq 2 .\end{cases}
\end{aligned}
$$

The estimate for the exceptional set that is implicit in this inequality is the one given by Theorem 2 in [10] for $n=1$ and by formula (1.7) in [6] for $n=2$, and is right in its dependence on $R$ whatever the value of $n$. To see this, let $\psi(z)=\sum_{n=1}^{\infty} a_{n} z^{n}$ be 
a transcendental entire function of one complex variable $z$. As we have shown, we have

$$
\left|a_{n}\right| r^{n} \leq \mu(r, \psi) \frac{\alpha_{n}}{\alpha_{N}} \rho_{N}^{n-N},
$$

where $N=N(r, \psi)$, for all $r$ outside an exceptional set $E$ of finite logarithmic measure. In fact the set $E$ has positive logarithmic measure: $E$ contains small open intervals about every point at which the central index changes value. For if $r_{0}$ is such a point, with, say,

$$
N^{\prime}=\lim _{r \rightarrow r_{0}^{-}} N(r, \psi)<\lim _{r \rightarrow r_{0}^{+}} N(r, \psi)=N^{\prime \prime},
$$

and if (53) held for a sequence of $r<r_{0}$ tending to $r_{0}$, we would have in particular

$$
\frac{\left|a_{N^{\prime \prime}}\right|}{\left|a_{N^{\prime}}\right|} r^{N^{\prime \prime}-N^{\prime}} \leq \frac{\left|\alpha_{N^{\prime \prime}}\right|}{\left|\alpha_{N^{\prime}}\right|} \rho_{N^{\prime}} N^{N^{\prime \prime}-N^{\prime}}
$$

for all $r<r_{0}$ close enough to $r_{0}$. Allowing $r$ to approach $r_{0}$ then,

$$
1 \leq \frac{\left|\alpha_{N^{\prime \prime}}\right|}{\left|\alpha_{N^{\prime}}\right|} \rho_{N^{\prime}} N^{\prime \prime}-N^{\prime}
$$

which contradicts (6). Thus (53) fails for all $r<r_{0}$ sufficiently close to $r_{0}$. A similar argument shows that (53) also fails for all $r>r_{0}$ sufficiently close to $r_{0}$. Thus the exceptional set for $f(\mathbf{z})=\psi\left(z_{1}\right)$ is $E \times \mathbb{R}^{n-1}$, and the part of this set in $\Delta_{\mathbf{1}, R \mathbf{1}}$ has logarithmic measure $(\log m e a s(E)+o(1))(\log R)^{n-1}$.

\section{Proof of Theorem 2}

Using (9) with $\mathbf{r}$ replaced by $\mathbf{R}$, and with $A_{\mathbf{N}}=\alpha_{\mathbf{N}}$ and $\mathbf{r}_{\mathbf{N}}=\boldsymbol{\rho}_{\mathbf{N}}$, we have

$$
\frac{\left|a_{\mathbf{j}}\right| \mathbf{r}^{\mathbf{j}}}{\left|a_{\mathbf{N}}\right| \mathbf{r}^{\mathbf{N}}}=\frac{\left|a_{\mathbf{j}}\right| \mathbf{R}^{\mathbf{j}}}{\left|a_{\mathbf{N}}\right| \mathbf{R}^{\mathbf{N}}}\left(\frac{\mathbf{r}}{\mathbf{R}}\right)^{\mathbf{j}-\mathbf{N}} \leq \frac{\alpha_{\mathbf{j}}}{\alpha_{\mathbf{N}}}\left(\frac{\mathbf{r} \boldsymbol{\rho}_{\mathbf{N}}}{\mathbf{R}}\right)^{\mathbf{j}-\mathbf{N}}
$$

and thus

$$
\sum_{\left|\mathbf{j}-\mathbf{N} \mathbf{1}_{\ell}\right| \geq \mathbf{k} \mathbf{1}_{\ell}} \mathbf{j}^{\mathbf{p}} \frac{\left|a_{\mathbf{j}}\right| \mathbf{r}^{\mathbf{j}}}{\left|a_{\mathbf{N}}\right| \mathbf{r}^{\mathbf{N}}} \leq \sum_{\left|\mathbf{j}-\mathbf{N} \mathbf{1}_{\ell}\right| \geq \mathbf{k} \mathbf{1}_{\ell}} \mathbf{j}^{\mathbf{p}} \frac{\alpha_{\mathbf{j}}}{\alpha_{\mathbf{N}}}\left(\frac{\mathbf{r} \boldsymbol{\rho}_{\mathbf{N}}}{\mathbf{R}}\right)^{\mathbf{j}-\mathbf{N}}=\prod_{l=1}^{n} S_{l \ell}
$$

where

$$
S_{l \ell}=\sum_{j_{l} \geq 0} j_{l}^{p_{l}} \frac{\alpha_{j_{l}}}{\alpha_{N_{l}}}\left(\frac{r_{l} \rho_{N_{l}}}{R_{l}}\right)^{j_{l}-N_{l}}, \quad l \neq \ell,
$$

and

$$
S_{\ell \ell}=\sum_{\left|j_{\ell}-N_{\ell}\right| \geq k_{\ell}} j_{\ell}^{p_{\ell}} \frac{\alpha_{j_{\ell}}}{\alpha_{N_{\ell}}}\left(\frac{r_{\ell} \rho_{N_{\ell}}}{R_{\ell}}\right)^{j_{\ell}-N_{\ell}} .
$$

The sums (56) and (57) are precisely those given by formulas (19) and (20) of [[7]]. According to the estimates obtained in the proof of [7, Theorem 2] - see, in particular, formulas (43), (45) and (51) of [7]-there is a number $N_{0}=N_{0}(\mathbf{p}, q, \epsilon, \beta)$ such that if $N_{*} \geq N_{0}$ then $S_{l \ell} \leq C N_{l}^{p_{l}+1}, l \neq \ell$, and $S_{\ell \ell} \leq C N_{\ell}^{p_{\ell}} N^{*-2 \beta}$, where $C=C(\mathbf{p})$. Thus, from (55),

$$
\sum_{\left|\mathbf{j}-\mathbf{N} \mathbf{1}_{\ell}\right| \geq \mathbf{k} \mathbf{1}_{\ell}} \mathbf{j}^{\mathbf{p}} \frac{\left|a_{\mathbf{j}}\right| \mathbf{r}^{\mathbf{j}}}{\left|a_{\mathbf{N}}\right| \mathbf{r}^{\mathbf{N}}} \leq C^{n} N^{* n-1-2 \beta} \mathbf{N}^{\mathbf{p}}
$$


which proves Theorem 2 .

\section{Proof of Theorem 3}

With $\gamma$ as in Theorem 3, we will apply Theorem 2 with

$$
\beta=\left(n-1+\|\mathbf{p}\|_{0}+\gamma\right) / 2 \text {. }
$$

In that case we have, from (16) and (23),

$$
\frac{\lambda_{j}}{N_{j}} \leq \frac{1}{2} \sqrt{\frac{N_{*}^{2} \alpha^{\prime}\left(N_{*}\right)}{N_{j}^{2} \alpha^{\prime}\left(N_{j}\right)}} \leq \frac{1}{2}, \quad 1 \leq j \leq n,
$$

if $\mathbf{N}$ is sufficiently large, since $t^{2} \alpha^{\prime}(t)$ is ultimately increasing and $N_{*} \rightarrow \infty$ as $\mathbf{R} \rightarrow \infty$, from (23). Thus, from (17), $\mathbf{k}=\boldsymbol{\lambda}$ whenever $\mathbf{N}$ is sufficiently large and so, from (59) and (16), we have

$$
\left\|\frac{\mathbf{k}}{\mathbf{N}}\right\| \leq \min \left\{\frac{1}{2}, \sqrt{\frac{30\left(n-1+\|\mathbf{p}\|_{\circ}+\gamma\right) \log N^{*}}{N_{*}^{2} \alpha^{\prime}\left(N_{*}\right)}}\right\}
$$

for all large $\mathbf{N}$. Let us note also that $\lambda_{*} \rightarrow \infty$ and therefore

$$
\|\mathbf{1} / \mathbf{k}\| \leq k_{*}^{-1}=\lambda_{*}^{-1} \rightarrow 0
$$

as $\mathbf{N} \rightarrow \infty$.

We write

$$
f(\mathbf{z})=\sum_{|\mathbf{j}-\mathbf{N}|<\mathbf{k}} a_{\mathbf{j}} \mathbf{z}^{\mathbf{j}}+\phi(\mathbf{z})=\mathbf{z}^{\mathbf{N}-\kappa} P(\mathbf{z})+\phi(\mathbf{z}),
$$

where $\boldsymbol{\kappa}=[\mathbf{k}]$ and $P$ is a polynomial of degree at most $2 \boldsymbol{\kappa}$, so that

$$
f^{(\mathbf{p})}(\mathbf{z})=\left(\mathbf{z}^{\mathbf{N}-\kappa} P(\mathbf{z})\right)^{(\mathbf{p})}+\phi^{(\mathbf{p})}(\mathbf{z}) .
$$

We have:

Lemma 6. With $\mathbf{R}, \mathbf{Z}$ and $\mathbf{z}$ as in the hypotheses of Theorem 3, and with $\mathbf{r}=|\mathbf{z}|$, the following hold as $\mathbf{R} \rightarrow \infty$ :

$$
\begin{aligned}
M(\mathbf{r}, P) & =\left(1+O\left(N^{*-\gamma}\right)\right) \mathbf{r}^{-\mathbf{N}+\boldsymbol{\kappa}} M(\mathbf{r}, f), \\
P(\mathbf{Z}) & =(1+o(1)) \mathbf{Z}^{-\mathbf{N}+\boldsymbol{\kappa}} f(\mathbf{Z}), \\
\left|P^{(\mathbf{j})}(\mathbf{z})\right| & \leq\left(\frac{2 e^{3} \boldsymbol{\kappa}}{\mathbf{R}}\right)^{\mathbf{j}} M(\mathbf{R}, P), \\
P(\mathbf{z}) & =(1+o(1)) P(\mathbf{Z}), \\
\left|\mathbf{z}^{\mathbf{p}} \phi^{(\mathbf{p})}(\mathbf{z})\right| & =o\left(\mathbf{r}^{\mathbf{N}-\boldsymbol{\kappa}}|P(\mathbf{z})|\right) .
\end{aligned}
$$

Proof. To prove Lemma 6, note first that (22) ensures that (18) holds if $\mathbf{R}$ is sufficiently large. Applying Theorem 2 with $\beta$ as in (58), we obtain

$$
\left|\mathbf{z}^{\mathbf{p}} \phi^{(\mathbf{p})}(\mathbf{z})\right| \leq C N^{* n-1+\|\mathbf{p}\|_{\circ}-2 \beta}\left|a_{\mathbf{N}}\right| \mathbf{r}^{\mathbf{N}} \leq C N^{*-\gamma}\left|a_{\mathbf{N}}\right| \mathbf{r}^{\mathbf{N}} \leq C N^{*-\gamma} M(\mathbf{r}, f) .
$$

With $\mathbf{p}=\mathbf{0}$ in (69), we have $|\phi(\mathbf{z})| \leq C N^{*-\gamma} M(\mathbf{r}, f)$, and (64) follows from this. Similarly, using (20) and (21), we have

$$
|\phi(\mathbf{Z})| \leq C N^{*-\gamma} M(\mathbf{R}, f)=C\|\mathbf{N}\|^{-\gamma} M(\mathbf{R}, f) \leq C\|\mathbf{N}\|^{-\gamma} \eta^{-1}|f(\mathbf{Z})|=o(|f(\mathbf{Z})|),
$$

and so we have (65). 
For (66) we need the following one-variable result [10, Lemma 7, p. 337]:

Suppose that $P(z)$ is a polynomial of degree $m$, and $|P(z)| \leq M$, for $|z| \leq r$. Then if $R \geq r$, we have

$$
\left|P^{\prime}(z)\right| \leq \frac{e M m R^{m-1}}{r^{m}}, \quad|z| \leq R
$$

Let $\mathbf{R}$ be large enough that $|\log (\mathbf{r} / \mathbf{R})| \leq \mathbf{k}^{-\mathbf{1}}$, which is possible from (22). If, for any $1 \leq \ell \leq n$, we take $r=R_{\ell}, R=R_{\ell} e^{1 / k_{\ell}}, m=2 \kappa_{\ell}$ and $M=M(\mathbf{R}, P)$ in (70), then we obtain

$$
\left|\frac{\partial P}{\partial z_{\ell}}\right| \leq \frac{2 e \kappa_{\ell}}{R_{\ell}} e^{\left(2 \kappa_{\ell}-1\right) / k_{\ell}} M(\mathbf{R}, P) \leq \frac{2 e^{3} \kappa_{\ell}}{R_{\ell}} M(\mathbf{R}, P)
$$

for $\left|z_{\ell}\right| \leq R_{\ell} e^{1 / k_{\ell}}$, using the fact that $\kappa_{\ell}=\left[k_{\ell}\right]$. Repeating the argument several times for each variable $z_{\ell}$, we obtain

$$
\left|P^{(\mathbf{j})}(\mathbf{z})\right| \leq\left(\frac{2 e^{3} \boldsymbol{\kappa}}{\mathbf{R}}\right)^{\mathbf{j}} M(\mathbf{R}, P)
$$

for $|\mathbf{z}| \leq \mathbf{R} e^{1 / \mathbf{k}}$, and (66) is this inequality restricted to the smaller set of $\mathbf{z}$ satisfying (22).

For (67), write $Q(\zeta)=P(\mathbf{Z}+(\mathbf{z}-\mathbf{Z}) \zeta)$, a function of one complex variable $\zeta$. Using the chain rule, $(71)$ and $(22)$, we have

$$
\left|Q^{\prime}(\zeta)\right| \leq\left|\left(\frac{\partial P}{\partial z_{1}}, \ldots, \frac{\partial P}{\partial z_{n}}\right) \cdot(\mathbf{z}-\mathbf{Z})\right| \leq 2 e^{3}\|\boldsymbol{\kappa}(\mathbf{z} / \mathbf{Z}-\mathbf{1})\|_{\circ} M(\mathbf{R}, P)
$$

for all large $\mathbf{R}$. From (22) and (61), we have $\mathbf{z} / \mathbf{Z} \rightarrow \mathbf{1}$ as $\mathbf{R} \rightarrow \infty$, so

$$
\|\boldsymbol{\kappa}(\mathbf{z} / \mathbf{Z}-\mathbf{1})\|_{\circ}=(1+o(1))\|\boldsymbol{\kappa} \log (\mathbf{z} / \mathbf{Z})\|_{\circ}=o(\eta)
$$

as $\mathbf{R} \rightarrow \infty$, again using (22). Thus $\left|Q^{\prime}(\zeta)\right|=o(\eta M(\mathbf{R}, P)$ and so

$$
|P(\mathbf{z})-P(\mathbf{Z})|=\left|\int_{0}^{1} Q^{\prime}(\zeta) d \zeta\right|=o(\eta M(\mathbf{R}, P))
$$

as $\mathbf{R} \rightarrow \infty$. From (65), (20) and (64), we have

$$
|P(\mathbf{Z})| \geq(1+o(1)) \eta M(\mathbf{R}, P),
$$

and this together with (73) gives (67).

For (68), we have, using in turn (67), (65), (20) and (64),

$$
\begin{aligned}
|P(\mathbf{z})| & =(1+o(1)) \mathbf{R}^{-\mathbf{N}+\boldsymbol{\kappa}} \mid f(\mathbf{Z}) \\
& \geq(1+o(1)) \eta \mathbf{R}^{-\mathbf{N}+\boldsymbol{\kappa}} M(\mathbf{R}, f)=(1+o(1)) \eta M(\mathbf{R}, P) .
\end{aligned}
$$

Also, from (72), $\mathbf{r}^{\kappa}=(1+o(1)) \mathbf{R}^{\boldsymbol{\kappa}}$. Thus, noting that $\boldsymbol{\kappa}=\mathbf{N}(\mathbf{R}, P)$ and using (75), we have

$$
\begin{aligned}
\left|a_{\mathbf{N}}\right| \mathbf{r}^{\mathbf{N}} & =\mathbf{r}^{\mathbf{N}-\kappa}\left|a_{\mathbf{N}}\right| \mathbf{r}^{\kappa}=O\left(\mathbf{r}^{\mathbf{N}-\kappa}\left|a_{\mathbf{N}}\right| \mathbf{R}^{\kappa}\right)=O\left(\mathbf{r}^{\mathbf{N}-\kappa} \mu(\mathbf{R}, P)\right) \\
& =O\left(\mathbf{r}^{\mathbf{N}-\kappa} M(\mathbf{R}, P)\right)=O\left(\eta^{-1} \mathbf{r}^{\mathbf{N}-\boldsymbol{\kappa}}|P(\mathbf{z})|\right),
\end{aligned}
$$

so that, from the middle inequality in (69), and (21),

$$
\left|\mathbf{z}^{\mathbf{p}} \phi^{(\mathbf{p})}(\mathbf{z})\right|=O\left(N^{*-\gamma} \eta^{-1} \mathbf{r}^{\mathbf{N}-\boldsymbol{\kappa}}|P(\mathbf{z})|\right)=o\left(\mathbf{r}^{\mathbf{N}-\kappa}|P(\mathbf{z})|\right) .
$$

This completes the proof of Lemma 6 . 
Returning to the proof of Theorem 3, we have

$$
\begin{aligned}
\mathbf{z}^{\mathbf{p}}\left(\mathbf{z}^{\mathbf{N}-\kappa} P(\mathbf{z})\right)^{(\mathbf{p})} & =\sum_{\mathbf{0} \leq \mathbf{j} \leq \mathbf{p}} \mathbf{C}_{\mathbf{j}}^{\mathbf{p}} \mathbf{z}^{\mathbf{p}}\left(\mathbf{z}^{\mathbf{N}-\boldsymbol{\kappa}}\right)^{(\mathbf{p}-\mathbf{j})} P^{(\mathbf{j})}(\mathbf{z}) \\
& =\sum_{\mathbf{0} \leq \mathbf{j} \leq \mathbf{p}} \mathbf{C}_{\mathbf{j}}^{\mathbf{p}} \frac{(\mathbf{N}-\boldsymbol{\kappa}) !}{(\mathbf{N}-\boldsymbol{\kappa}-\mathbf{p}+\mathbf{j}) !} \mathbf{z}^{\mathbf{N}-\boldsymbol{\kappa}+\mathbf{j}} P^{(\mathbf{j})}(\mathbf{z})
\end{aligned}
$$

where

$$
\mathbf{C}_{\mathbf{j}}^{\mathbf{p}}=\prod_{i=1}^{n} C_{j_{i}}^{p_{i}} \quad \text { and } \quad \frac{(\mathbf{N}-\boldsymbol{\kappa}) !}{(\mathbf{N}-\boldsymbol{\kappa}-\mathbf{p}+\mathbf{j}) !}=\prod_{i=1}^{n} \frac{\left(N_{i}-\kappa_{i}\right) !}{\left(N_{i}-\kappa_{i}-p_{i}+j_{i}\right) !} .
$$

Here $C_{j_{i}}^{p_{i}}$ is the usual binomial coefficient. The term on the right-hand side of (77) that corresponds to $\mathbf{j}=\mathbf{0}$ is

$$
\frac{(\mathbf{N}-\boldsymbol{\kappa}) !}{(\mathbf{N}-\boldsymbol{\kappa}-\mathbf{p}) !} \mathbf{z}^{\mathbf{N}-\boldsymbol{\kappa}} P(\mathbf{z})=(1+\sigma) \mathbf{N}^{\mathbf{p}} \mathbf{z}^{\mathbf{N}-\boldsymbol{\kappa}} P(\mathbf{z}),
$$

where, making use of the first half of (60),

$$
|\sigma| \leq C\left\|\frac{\mathbf{k}}{\mathbf{N}}\right\|
$$

and $C=C(\mathbf{p})$.

We proceed to estimate the remaining terms in (77). From (66) and (75),

$$
\left|P^{(\mathbf{j})}(\mathbf{z})\right| \leq(1+o(1)) \eta^{-1}\left(2 e^{3} \boldsymbol{\kappa} / \mathbf{r}\right)^{\mathbf{j}}|P(\mathbf{z})| .
$$

It follows that any term in (77) corresponding to a value of $\mathbf{j} \neq \mathbf{0}$ is

$$
\begin{aligned}
O\left(\mathbf{N}^{\mathbf{p}-\mathbf{j}} \mathbf{r}^{\mathbf{N}-\boldsymbol{\kappa}+\mathbf{j}}\left|P^{(\mathbf{j})}(\mathbf{z})\right|\right) & =O\left(\eta^{-1}\left(2 e^{3} \boldsymbol{\kappa} / \mathbf{N}\right)^{\mathbf{j}} \mathbf{N}^{\mathbf{p}} \mathbf{r}^{\mathbf{N}-\boldsymbol{\kappa}}|P(\mathbf{z})|\right) \\
& =O\left(\eta^{-1} \sigma \mathbf{N}^{\mathbf{p}} \mathbf{r}^{\mathbf{N}-\boldsymbol{\kappa}}|P(\mathbf{z})|\right),
\end{aligned}
$$

where $\sigma$ satisfies (80). From this and (79), we have

$$
\mathbf{z}^{\mathbf{p}}\left(\mathbf{z}^{\mathbf{N}-\kappa} P(\mathbf{z})\right)^{(\mathbf{p})}=\left(1+O\left(\eta^{-1} \sigma\right)\right) \mathbf{N}^{\mathbf{p}} \mathbf{z}^{\mathbf{N}-\kappa} P(\mathbf{z})
$$

and thus, using (68),

$$
\mathbf{z}^{\mathbf{p}} f^{(\mathbf{p})}(\mathbf{z})=\left(1+O\left(\eta^{-1} \sigma\right)+o(1)\right) \mathbf{N}^{\mathbf{p}} \mathbf{z}^{\mathbf{N}-\kappa} P(\mathbf{z}) .
$$

Taking $\mathbf{p}=\mathbf{0}$ in (68), we deduce that $f(\mathbf{z})=(1+o(1)) \mathbf{z}^{\mathbf{N}-\boldsymbol{\kappa}} \mathbf{P}(\mathbf{z})$, and Theorem 3 follows.

\section{Proof of Theorem 4}

For (a), we have $\boldsymbol{\Phi}\left(\left(\mathbf{z}^{\mathbf{1}}\right)^{-1 /(n+1)} \mathbf{z}\right)=\mathbf{z}$. Also, if $\boldsymbol{\Phi}\left(\boldsymbol{\zeta}_{1}\right)=\boldsymbol{\Phi}\left(\boldsymbol{\zeta}_{2}\right)$ then $\boldsymbol{\Phi}\left(\boldsymbol{\zeta}_{1}\right)^{\mathbf{1}}=$ $\boldsymbol{\Phi}\left(\boldsymbol{\zeta}_{2}\right)^{\mathbf{1}}$; that is, $\left(\boldsymbol{\zeta}_{1}^{\mathbf{1}}\right)^{n+1}=\left(\boldsymbol{\zeta}_{2}^{\mathbf{1}}\right)^{n+1}$ and so $\boldsymbol{\zeta}_{1}^{\mathbf{1}}=\boldsymbol{\zeta}_{2}^{\mathbf{1}}$, if $\boldsymbol{\zeta}_{1}, \boldsymbol{\zeta}_{2}>\mathbf{0}$. Thus $\boldsymbol{\zeta}_{1}=$ $\left.\left.\Phi\left(\zeta_{1}\right)\right) / \zeta_{1}^{1}=\Phi\left(\zeta_{2}\right)\right) / \zeta_{2}^{1}=\zeta_{2}$. 
Concerning the Jacobian, we have, subtracting each row from the one before and adding each column to the one after,

$$
\begin{aligned}
\frac{\partial(\log \mathbf{z})}{\partial(\log \zeta)} & =\left|\begin{array}{cccccc}
2 & 1 & 1 & \cdots & 1 & 1 \\
1 & 2 & 1 & \cdots & 1 & 1 \\
\vdots & \vdots & \vdots & & \vdots & \vdots \\
1 & 1 & 1 & \cdots & 2 & 1 \\
1 & 1 & 1 & \cdots & 1 & 2
\end{array}\right|=\left|\begin{array}{ccccccc}
1 & -1 & 0 & \cdots & 0 & 0 \\
0 & 1 & -1 & \cdots & 0 & 0 \\
\vdots & \vdots & \vdots & & \vdots & \vdots \\
0 & 0 & 0 & \cdots & 1 & -1 \\
1 & 1 & 1 & \cdots & 1 & 2
\end{array}\right| \\
& =\left|\begin{array}{cccccc}
1 & 0 & 0 & \cdots & 0 & 0 \\
0 & 1 & 0 & \cdots & 0 & 0 \\
\vdots & \vdots & \vdots & & \vdots & \vdots \\
0 & 0 & 0 & \cdots & 1 & 0 \\
1 & 2 & 3 & \cdots & n-1 & n+1
\end{array}\right|=n+1 .
\end{aligned}
$$

For (b), we have $\boldsymbol{\Phi}(\boldsymbol{\zeta})^{\mathbf{j}}=\boldsymbol{\zeta}^{\mathbf{j}+\|\mathbf{j}\|_{0} \mathbf{1}}$, so that if $\operatorname{deg}\left(\boldsymbol{\Phi}(\boldsymbol{\zeta})^{\mathbf{j}_{1}}\right)=\operatorname{deg}\left(\boldsymbol{\Phi}(\boldsymbol{\zeta})^{\mathbf{j}_{2}}\right)$ then $\mathbf{j}_{\mathbf{1}}+\left\|\mathbf{j}_{\mathbf{1}}\right\|_{\circ} \mathbf{1}=\mathbf{j}_{\mathbf{2}}+\left\|\mathbf{j}_{\mathbf{2}}\right\|_{\text {。 }} \mathbf{1}$. Summing the components of each side, we obtain $\left\|\mathbf{j}_{\mathbf{1}}\right\|_{\circ}=$ $\left\|\mathbf{j}_{\mathbf{2}}\right\|_{\text {。 }}$ and therefore $\mathbf{j}_{\mathbf{1}}=\mathbf{j}_{\mathbf{2}}$.

Concerning (c), note that, from (b), the Taylor series for $\mathcal{F}(\boldsymbol{\zeta})$ is $\sum_{\mathbf{j} \geq \mathbf{0}} a_{\mathbf{j}} \boldsymbol{\Phi}(\boldsymbol{\zeta})^{\mathbf{j}}$. It follows that $\mu(\boldsymbol{\zeta}, \mathcal{F})=\mu(\boldsymbol{\Phi}(\boldsymbol{\zeta}), f)$ for $\boldsymbol{\zeta}>\mathbf{0}$ and also, with $\mathbf{N}=\mathbf{N}(\boldsymbol{\Phi}(\overline{\boldsymbol{\zeta}}), f)$, that $\mathbf{N}(\zeta, \mathcal{F})=\operatorname{deg}\left(\boldsymbol{\Phi}(\zeta)^{\mathbf{N}}\right)=\mathbf{N}+\|\mathbf{N}\|_{0} 1$.

If $\boldsymbol{\zeta}_{0}$ is such that $\left|\boldsymbol{\zeta}_{0}\right|=\boldsymbol{\zeta}$ and $\left|\mathcal{F}\left(\boldsymbol{\zeta}_{0}\right)\right|=M(\boldsymbol{\zeta}, \mathcal{F})$, then $\left|\boldsymbol{\Phi}\left(\boldsymbol{\zeta}_{0}\right)\right|=\boldsymbol{\Phi}(\boldsymbol{\zeta})$ and thus $M(\boldsymbol{\zeta}, \mathcal{F})=\left|f\left(\boldsymbol{\Phi}\left(\boldsymbol{\zeta}_{0}\right)\right)\right| \leq M(\boldsymbol{\Phi}(\boldsymbol{\zeta}), f)$. Conversely, suppose that $\mathbf{z}_{\mathbf{0}}$ is such that $\left|\mathbf{z}_{\mathbf{0}}\right|=\boldsymbol{\Phi}(\boldsymbol{\zeta})$ and $\left|f\left(\mathbf{z}_{\mathbf{0}}\right)\right|=M(\boldsymbol{\Phi}(\boldsymbol{\zeta}), f)$. Let $\boldsymbol{\zeta}_{0}>\mathbf{0}$ be such that $\mathbf{z}_{\mathbf{0}}=\boldsymbol{\Phi}\left(\boldsymbol{\zeta}_{0}\right)$; $\boldsymbol{\zeta}_{0}$ exists from (a). We have $\left|\boldsymbol{\Phi}\left(\boldsymbol{\zeta}_{0}\right)\right|=\boldsymbol{\Phi}\left(\left|\boldsymbol{\zeta}_{0}\right|\right)$ and so, from (a), $\left|\boldsymbol{\zeta}_{0}\right|=\boldsymbol{\zeta}$. Hence $M(\boldsymbol{\Phi}(\boldsymbol{\zeta}), f)=\left|f\left(\mathbf{z}_{0}\right)\right|=\left|f\left(\Phi\left(\boldsymbol{\zeta}_{0}\right)\right)\right|=\left|\mathcal{F}\left(\boldsymbol{\zeta}_{0}\right)\right| \leq M(\boldsymbol{\zeta}, \mathcal{F})$. Combining the two inequalities we obtain $M(\boldsymbol{\zeta}, \mathcal{F})=M(\boldsymbol{\Phi}(\boldsymbol{\zeta}), f)$.

Finally, (d) follows from Theorem 3 since, from the last part of (c), we have $\mathcal{N}^{*} \leq 2 \mathcal{N}_{*}$, and thus (23) is trivially satisfied.

\section{An application of Theorem 4}

The method developed in [8] and sketched here is this: given a partial differential equation for an entire function $f(\mathbf{z})$, rewrite the equation in terms of $\mathcal{F}$ and $\boldsymbol{\zeta}$, given by (30) and (29), make use of (41) and then express the conclusions, whatever they are, in terms of $f$. The conclusion in the example that we consider below, and it appears to be typical, provides a lower bound for the lower order of the solution; here, as usual, the order and lower order of $f$ are the upper and lower limits of

$$
\frac{\log \log M(r \mathbf{1}, f)}{\log r}
$$

as $r \rightarrow \infty$. The key points in the method are contained in the following lemma.

Lemma 7. With $\boldsymbol{\zeta}$ as in (29), we have

$$
(n+1) \frac{\partial \zeta_{i}}{\partial z_{j}}= \begin{cases}-\zeta_{i} / z_{j}, & i \neq j, \\ n \zeta_{i} / z_{j}, & i=j .\end{cases}
$$

Also, with $\mathcal{F}$ as in (30),

$$
\|\mathbf{N}(\mathcal{R}, \mathcal{F})\| \leq \log M(\mathcal{R}, \mathcal{F})(\log \log M(\mathcal{R}, \mathcal{F}))^{2}
$$


for all $\mathcal{R}$ that are either

normal for $\mathcal{F}$ with respect to $h_{\alpha}$ (given by (7), where $\alpha$ is given by (12) for some $\epsilon$ and $q)$, if $\mathcal{F}(\mathbf{0}) \neq 0$, or

normal for $\mathcal{F}+1$ with respect to $h_{\alpha}$, if $\mathcal{F}(\mathbf{0})=0$,

and for which $\mathbf{N}(\mathcal{R}, \mathcal{F})$ is sufficiently large.

Proof. For the first part of Lemma 7 we have, from $(29), \mathbf{z}^{\mathbf{1}}=\left(\zeta^{\mathbf{1}}\right)^{n+1}$ and therefore $\boldsymbol{\zeta}=\mathbf{z}^{-\mathbf{1} /(n+1)} \mathbf{z}$. Taking logarithms of both sides and differentiating, we obtain (84).

For the second part, we use Remarks (b) and (c) following Theorem 1 Corollary 1. Suppose first that $\mathcal{F}(\mathbf{0}) \neq 0$ and that $\mathcal{R}$ is normal for $\mathcal{F}$. Taking $\mathbf{j}=\mathbf{0}$ in (9), we have

$$
\log \left(\alpha_{\mathcal{N}} \boldsymbol{\rho}_{\mathcal{N}}^{\mathcal{N}}\right) \leq \log (\mu(\boldsymbol{R}, \mathcal{F}) / \mathcal{F}(\mathbf{0}))
$$

where $\mathcal{N}=\mathbf{N}(\mathcal{R}, \mathcal{F})$. As in [[6], formula (4.7)], the left-hand side of (86) is

$$
(1+o(1)) \sum_{j=1}^{n} \frac{\mathcal{N}_{j}}{\log \mathcal{N}_{j} \log _{2} \mathcal{N}_{j} \cdots\left(\log _{q+1} \mathcal{N}_{j}\right)^{1+\epsilon}}
$$

as $\mathcal{N} \rightarrow \infty$, recalling that, from $(32), \mathcal{N}_{j} \rightarrow \infty$ for each $j=1, \ldots, n$ as $\mathcal{N} \rightarrow \infty$. It follows that

$$
\frac{\mathcal{N}}{\log \mathcal{N} \log _{2} \mathcal{N} \cdots\left(\log _{q+1} \mathcal{N}\right)^{1+\epsilon}} \leq(1+o(1)) \log (\mu(\mathcal{R}, \mathcal{F}) / \mathcal{F}(\mathbf{0}))
$$

Thus $\log \|\mathcal{N}\| \leq(1+o(1)) \log _{2} \mu(\mathcal{R}, \mathcal{F})$ as $\mathcal{N} \rightarrow \infty$ and therefore

$$
\begin{aligned}
\|\mathcal{N}\| & \leq(1+o(1)) \log (\mu(\mathcal{R}, \mathcal{F}) / \mathcal{F}(\mathbf{0})) \log \|\mathcal{N}\| \log _{2}\|\mathcal{N}\| \cdots\left(\log _{q+1}\|\mathcal{N}\|\right)^{1+\epsilon} \\
& \leq \log \mu(\mathcal{R}, \mathcal{F})\left(\log _{2} \mu(\mathcal{R}, \mathcal{F})\right)^{2} \leq \log M(\mathcal{R}, \mathcal{F})\left(\log _{2} M(\mathcal{R}, \mathcal{F})\right)^{2}
\end{aligned}
$$

for all $\mathcal{R}$ that are normal for $\mathcal{F}$ and such that $\mathcal{N}$ is sufficiently large.

If $\mathcal{F}(\mathbf{0})=\mathbf{0}$, we consider instead $\mathcal{G}=\mathcal{F}+1$ and suppose that $\mathcal{R}$ is normal for $\mathcal{G}$. As above we obtain $\|\mathbf{N}(\mathcal{R}, \mathcal{G})\| \leq \log \mu(\boldsymbol{\mathcal { R }}, \mathcal{G})\left(\log _{2} \mu(\boldsymbol{\mathcal { R }}, \mathcal{G})\right)^{2}$ if $\mathbf{N}(\boldsymbol{\mathcal { R }}, \mathcal{G})$ is sufficiently large. Then (85) follows since, as we noted in Remark (c) following Theorem 1 Corollary $1, \mu(\mathcal{R}, \mathcal{G})=\mu(\mathcal{R}, \mathcal{F})$ and $\mathbf{N}(\mathcal{R}, \mathcal{G})=\mathbf{N}(\mathcal{R}, \mathcal{F})$ for any $\mathcal{R}$ for which $\mu(\mathcal{R}, \mathcal{F})>1$; and this condition is satisfied if $\mathbf{N}(\mathcal{R}, \mathcal{F})$ is sufficiently large, from (87). This completes the proof of Lemma 7.

Remark. Higher derivatives of $\zeta_{i}$ can be obtained from (84) by repeated differentiation.

To illustrate the method, consider the differential equation

$$
\frac{\partial f}{\partial z_{1}}+\cdots+\frac{\partial f}{\partial z_{n}}=f
$$

for which $E(\mathbf{z})$ given by $(27)$ is a solution. As we noted previously, Theorem 3 is not applicable to $E$.

We write $f(\mathbf{z})=\mathcal{F}(\boldsymbol{\zeta})$, where $z=\boldsymbol{\Phi}(\boldsymbol{\zeta})$ as in (29), and obtain, from the first part of Lemma 7 ,

$$
\left|\frac{\partial f}{\partial z_{j}}\right|=\left|\sum_{i=1}^{n} \frac{\partial \mathcal{F}}{\partial \zeta_{i}} \frac{\partial \zeta_{i}}{\partial z_{j}}\right| \leq \sum_{i=1}^{n}\left|\frac{\partial \mathcal{F}}{\partial \zeta_{i}}\right|\left|\frac{\zeta_{i}}{z_{j}}\right|
$$


From (89) then,

$$
|\mathcal{F}| \leq \sum_{j=1}^{n} \sum_{i=1}^{n}\left|\frac{\partial \mathcal{F}}{\partial \zeta_{i}}\right|\left|\frac{\zeta_{i}}{z_{j}}\right|
$$

Given $\mathcal{R}>0$, let $\mathcal{R}$ be normal, either for $\mathcal{F}$ with respect to $h_{\alpha}$ if $\mathcal{F}(\mathbf{0}) \neq \mathbf{0}$, or for $\mathcal{F}+1$ with respect to $h_{\alpha}$ if $\mathcal{F}(\mathbf{0})=\mathbf{0}$, and such that $\mathcal{R} \mathbf{1}<\mathcal{R}<\mathcal{R}^{1+2 \epsilon} \mathbf{1}$. Here $\epsilon$ is the number occurring in the definition of $\alpha$. From (13), such an $\mathcal{R}$ exists. Let $\boldsymbol{\zeta}$ be such that $|\boldsymbol{\zeta}|=\mathcal{R}$ and $|\mathcal{F}(\boldsymbol{\zeta})|=M(\mathcal{R}, \mathcal{F})$. In the case $\mathcal{F}(\mathbf{0})=\mathbf{0}$, notice that, if $c$ is such that $|c|=1$ and $\arg c=\arg \mathcal{F}(\boldsymbol{\zeta})$, then $\mathcal{R}$ is normal for $\mathcal{F}+c$ and $|\mathcal{F}(\boldsymbol{\zeta})+c|=M(\mathcal{R}, \mathcal{F}+c)$. Applying (41) to $\mathcal{F}$ or $\mathcal{F}+c$ as appropriate, we obtain

$$
\frac{\partial \mathcal{F}}{\partial \zeta_{i}}(\boldsymbol{\zeta})=(1+o(1)) \frac{\mathcal{N}_{i}}{\zeta_{i}} \mathcal{F}(\boldsymbol{\zeta})
$$

where $\mathcal{N}_{i}$ is the $i$ th component of $\mathcal{N}=\mathcal{N}(\mathcal{R}, \mathcal{F})$, and substituting this into (90) we have

$$
1 \leq(1+o(1)) \sum_{j=1}^{n} \sum_{i=1}^{n} \frac{\mathcal{N}_{i}}{\left|z_{j}\right|} \leq(n+o(1))\|\mathcal{N}\| \sum_{j=1}^{n} \frac{1}{\left|z_{j}\right|}
$$

Now

and so

$$
\left|z_{j}\right|=\left|\zeta_{1}\right| \ldots\left|\zeta_{j}\right|^{2} \ldots\left|\zeta_{n}\right| \geq \mathcal{R}^{n+1}
$$

$$
\left(n^{-2}+o(1)\right) \mathcal{R}^{n+1} \leq\|\mathcal{N}\| .
$$

From the second part of Lemma 7 then,

$$
\begin{aligned}
\left(n^{-2}+o(1)\right) \mathcal{R}^{n+1} & \leq \log M(\mathcal{R}, \mathcal{F})(\log \log M(\mathcal{R}, \mathcal{F}))^{2} \\
& \leq \log M\left(\mathcal{R}^{1+2 \epsilon} \mathbf{1}, \mathcal{F}\right)\left(\log \log M\left(\mathcal{R}^{1+2 \epsilon} \mathbf{1}, \mathcal{F}\right)\right)^{2}
\end{aligned}
$$

from which it follows that $\mathcal{F}$ has lower order at least $(n+1) /(1+2 \epsilon)$. Since this holds for any $\epsilon>0, \mathcal{F}$ has lower order at least $n+1$. Also, from $(31), M(\mathcal{R} \mathbf{1}, \mathcal{F})=$ $M\left(\mathcal{R}^{n+1} \mathbf{1}, f\right)$ and we conclude that the lower order of $f$ is at least 1 . Note that $E$ has lower order (and order) 1.

\section{References}

[1] Bergweiler, W.: The size of Wiman-Valiron discs. - Complex Var. Elliptic Equ. 56, 2011, $13-33$.

[2] Clunie, J.: The determination of an integral function of finite order by its Taylor series. - J. London Math. Soc. 28, 1953, 58-66.

[3] Clunie, J.: On the determination of an integral function from its Taylor series. - J. London Math. Soc. 30, 1955, 32-42.

[4] Fenton, P.C.: Wiman-Valiron theory for entire functions of finite lower growth. - Trans. Amer. Math. Soc. 252, 1979, 221-232.

[5] Fenton, P. C.: A note on the Wiman-Valiron method. - Proc. Edinb. Math. Soc. 37, 1994, $53-55$.

[6] Fenton, P. C.: Wiman-Valiron theory in two variables. - Trans. Amer. Math. Soc. 347, 1995, 4403-4412.

[7] Fenton, P. C.: Polynomial approximation of entire functions of two variables. - Complex Var. Theory Appl. 40, 2000, 211-230. 
[8] Fenton, P. C., and J. Rossi: Two-variable Wiman-Valiron theory and PDEs. - Ann. Acad. Sci. Fenn. Math. 35, 2010, 571-580.

[9] Hayman, W. K.: A generalisation of Stirling's formula. - J. Reine Angew. Math. 196, 1956, $67-95$.

[10] Hayman, W. K.: The local growth of power series: a survey of the Wiman-Valiron method. - Canad. Math. Bull. 17, 1974, 317-358.

[11] Kövari, T.: On theorems of G. Pólya and P. Turán. - J. Anal. Math. 6, 1958, 323-332.

[12] Kövari, T.: On the Borel exceptional values of lacunary integral functions. - J. Anal. Math. 9, 1961/1962, 71-109.

[13] Valiron, G.: Lectures on the general theory of integral functions. - Chelsea (New York), 1949.

Received 18 October 2011 • Accepted 27 August 2012 